\title{
EXPERIMENTAL AND COMPUTATIONAL STUDIES \\ OF POLAR SOLVATION
}

DE-FG02-89ER14020

$\operatorname{Dec} / \operatorname{te} / / 4(12)-3$

DOE/ER/ $14020-3$

DE92 017298

\section{RD YEAR PROGRESS REPOR'T}

During the years 1989-1991 the following articles were published as a result of research supported by our initial DOE grant "Experimental and Computational Studies of Polar Solvation":

1. M. Maroncelli, J. Maclnnis, and G. R. Fleming, "Polar Solvent Dynamics and Electron Transfer Reactions," Science 243, 1674-1681 (1989).

i. R. S. Fee, C. F. Chapman, and M. Maroncelli, "Solvation Dynamics in N-Methyl Amides,"J. Phys. Chem. 94, 4929-4935 (1990).

3. M. Maroncelli, R. S. Fee, C. F. Chapman, and G. R.Fleming, "Dynamic Stokes Shift in Coumarin: Is It Only Relaxation?," J. Phys. Chem. 95, 1012-1014 (1991).

4. M. Maroncelli, "Computer Simulations of Solvation Dynamics in Acetonitrile," J. Chem. Phys. 94, 2084-2103 (1.991).

5. R. S. Fee, J. A. Milson, and M. Maroncelli, "Time - Dependent Fluorescence Stokes Shifts at Low Temperatures: Non-Dynamical Mechanisms," J. Phys. Chem. 95, 5170)-5181 (1991).

6*. C. F. Chapman and M. Maroncelli, "Fluorescence Studies of Solvation and Solvation Dynamics in Ionic Solutions," J. Phys. Chem. 95, 9095.9114 (1991).

7*. R. S. Moog and M. Maroncelli, "7-Azaindole in Alcohols: Solvation Dynamics and Proton Transfer," J. Phys. Chem. 95, 10359-10368 (1991).

8*. M. Maroncelli, "The Dynamics of Polar Solvation," J. Mol. Liquids 50, 0000 (1991).

9*. A. Papazyan and M. Maroncelli, "Simulations of Solvation in a Brownian Dipole Lattice," J. Chem. Phys. 95, 0000 (1991).

We have also completed the experimental work for two other papers which are presently in rough draft form:

10. R. S. Fee and M. Maroncelli, "Determining Time Zero in Time Evolving Spectra," manuscript in preparation.

11. R. S. Moog, D. L. Bankert, and M. Maroncelli, "Dielectric Friction and the Rotational Dynamics of Coumarin 102 - Alcohol Complexes, manuscript in
preparation.

A number of projects that are still underway are described in our renewal proposal. Below we provide abstracts and a brief commentary on the content of the articles listed above. In addition, several of them have been enclosed along with this report; these are marked by an asterisk. 
The following two review articles describe our general interest in solvation dynamics $[1,8]$ and the relationship of such dynamics to charge transter reactions [1]. The second of these [8] is especially relevant to the present report since it is an up to date summary of the field of solvation dynamics and also contains a number of origiral results of our work not yet published elsewhere.

1. "Polar Solvent Dynamics and Electron-Transfer Reactions", M. Maroncelli, J. Macinnis, G. R. Fleming, Science 243, 1674"1081 (1989).

Polar solvents often exert a dramatic influence on reactions in solution. Equilibrium aspects of this influence involve differential solvation of reactants compared to the transition state that lead to alteration of the free-energy barrier to reaction. Such effects are well known, and often give rise changes in reaction rates of many orders of magnitude. Less well understood are effects arising from non-equilibrium, dynamical aspects of solvation. During the course of reaction, charge is rapidly redistributed among reactants. How the reaction couples to its solvent environment depends critically on how fast the solvent can respond tis these changes in reactant charge distribution. In this article the dynamics of solvation in polar liquids and the influence of this dynamics on electron-transfer reactions are discussed. A molecular picture suggests that polar solvation occurs on multiple time scales as a result of the involvement of different types of solvent motion. A hierarchy of models from a homogeneous continuum model to one incorporating molecular aspects of solvation, combined with computer simulations, gives insight into the underlying dynamics. Experimental measures of solvation dynamics from picosecond and subpicosecond time-dependent Stokes shift studies are compared with the predictions of theoretical models. The implication of these results for electron-transfer reaction in solution are then briefly considered.

8. "The Dynamics of Solvation in Polar Liquics", M. Maroncelli, J. Mol. Liquids, submitted June 1991.

Recent experiments, theories, and computer simulations aimed at elucidating the dynamics of solvation on polar liquids are critically reviewed.

We have completed several experimental studies of related to solvation and solvation dynamics in pure polar solvents $[2,3,5]$. Reference 2 concerns the dynamics of a class of solvents, the $\mathrm{N}$-methyl arnides, that were originally though to show very large deviations from continum predictions. It turns out that, like most other solvents, the $\mathrm{N}$-methyl amides in fact do show continuum-like solvation times [see $\mathrm{M}$. Maroncelli and G. R. Fleming, J. Chem. Phys. 92, 3251 (1990) for an explanation of why our original work came to a different conclusion]. Paper \#2 explains why the continuum prediction holds good in these highly associated solvents even though molecular theories predict otherwise. References 3 and 5 deal with two new and general effects that arise in the spectroscopy of highly soivent sensitive solutes.

2. "Solvation Dynamics in N-Methyl Amides," C.F. Chapman, R. S. Fee, and M. Maroncelli, J. Phys. Chem. 94.4929.4935 (1990).

Solvation times in three homologous amides, N-methylformamide, N.methylacetamide, and N.methylpropionamide have been determined from measurements of the dynamic Stokes shift of the fluorescence spectra of two probe solutes, "prodan" and coumarin 102. Single particle reorientation times have also been measured in one of these solvents, N-methylfornamide using NMR methods. The solvation dynamics are compared to two theoretical models, the simple continuum model and the dynamical MSA model. Although neither model predicts the time dependence of the response satisfactorily, the average solvation times observed are close to the solvent longitudinal relaxation time $\left(\tau_{L}\right)$ predicted by the simple continuum model. In contrast, the predictions of the dynamical MSA model are approximately four times slower than the observed solvation 
response. The failure of the latter model appear, to result from an over-estimation of the single-particle reorientation times of these solvents. Estimates of such single particle times based on the NMR measurements are within a factor of two of $\tau_{\mathrm{L}}$. This similarity seems to account for the near equality of average solvation times and $\tau_{L}$ in the amides.

3. "Dynamic Stokes Shift in Coumarin: Is It Only Relaxation?", $M$. Maroncelli, R. S. Fee, C. F. Chapman, and G. R. Fleming, J. Phys. Chem 95, 1012.1014 (1991).

Recently, Agmon [J. Phys. Chem. 94, 2959 (1990)] has raised important questions regarding the mechanism of the time-dependent fluorescence Stokes shift in polar solvents. On the basis of the non-exponential decay of total fluorescence observed in coumarin 153 he proposes that the observed spectral dynamics may be related to an inhomogeneous decay mechanism rather than to solvent relaxation. We show here that the non-exponential fluorescence decays can be quantitatively understood in terms of time-dependent changes in the radiative rate brought about by the well-known $v^{3}$ dependence of radiative rate on emission frequency. We conclude that the operation of the inhomogeneous mechanism proposed by Agmon probably has a very small impact on the spectral dynamics measured to date.

5. "Inhomogeneous Decay Kinetics and Apparent Solvent Relaxation at Low Temperatures", R. S. Fee, J. A. Milsom, and M. Maroncelli, J. Phys. Chem, (1991).

We have observed time-dependent shifts in the fluorescence spectra of solvatochromic probe molecules in frozen polar solvents. The phenomenon is a general one, and is observed in a variety of fluorophores in both hydrogen-bonding and non-hydrogen-bonding solvents. These shifts are not related to solvent relaxation but rather result from inhomogeneous fluorescence decay kinetics. Molecules in different portions of the fluorescence spectrum decay at different rates and thereby cause the spectrum to evolve in time due to non-uniform loss of excited state population. We have modeled such kinetics based on the assumption that fluorescence decay rates are solvent sensitive only through the relation between the radiative rate and emission frequency, $k_{\mathrm{rad}} \propto \mathrm{v}^{3}$. Measurements of the effect of excitation wavelength on steady-state fluorescence spectra are used to quantify the inhomogeneous broadening present in a number of fluorophore/solvent systems. These results are then used as input to model their time-dependence. In most cases, nearly quantitative agreement between the calculated and observed spectral dynamics is achieved.

We have extended our study of solvation dynamics to include ionic solutions [6]. Based on measurements of a broad range of ionic solutions and fluorescence probes we conclude that the dynamics in ionic solution are best understood in terms of a sequential complexation model. These dynamics are completely different in nature from prior expectations of how ion atmosphere relaxation occurs.

6. "Fluorescence Studies of Ionic Solution Dynamics", C. F. Chapman and M. Maroncelli, J. Phys. Chem 95, 9095-9114 (1991)

We have employed steady-state and time-resolved emission spectroscopy to study static and dynamic aspects of the solvation of polar aromatic solutes in ionic solution. Several cormmon solvatochromic probe molecules (Cu102, Cu153, Prodan, 4-A.P) were examined in a wide range of ionic solutions consisting of a variety of salts (mainly $\mathrm{Li}^{+}, \mathrm{Na}^{+}, \mathrm{Mg}^{2+}, \mathrm{Ca}^{2+}, \mathrm{Sr}^{2+}, \mathrm{Ba}^{2+}$ perchlorates) in a number of non-aqueous solvents (tetrahydrofuran, acetone, propylene carbonate, acetonitrile, dimethylformarnide, dimethylsulfoxide, methanol, 1-propanol, and 
formamide). The presence of ions causes shifts in the spectra of these probe solutes similar to those observed in pure solvents of varying polarity. As a function of increasing salt concentration or time, the primary spectral change is a frequency shift with little accompanying change in the spectral shape or width. Ion-induced frequency shifts in steady-state spectra are typically in the range of several hundred $\mathrm{cm}^{-1}$ in $1 \mathrm{M}$ salt solutions. The magnitudes of these shifts decrease as the strength of solvent-solute interactions increases. They depend little on the identity of the anion but are approximately proportional to the charge-to-size ratio of the cation of the salt considered. The ionic solvation dynamics measured by tirne-resolved fluorescence take place on a 1-10 ns time scale. The kinetics is significantly dependent on excitation wavelength, especially for excitation on the red edge of the absorption spectrum. The rate of spectral shift is proportional to salt concentration and iatversely proportional to viscosity for concentrations of $1 \mathrm{M}$ or less. When these two factors are accounted for, the observed solvation rates are found to decrease with solvent polarity and decrease with the charge-to-size ratio of the cation. A number of the above results are inconsistent with the commonly used description of solute-ion interactions in terms of a diffuse ion-atmosphere. To rationalize our observations we propose a model oased on equilibrium among a limited set of solvates distinguished b: the number of cations in the first solvation shell of the probe.

We have performed two studies of solvation dynamics using computer simulation techniques. One employed a fairly realistic representation of acetonitrile [4] and the other a rather idealized model solvent consisting of a Brownian dipole lattice [9]. Both studies served to significantly enhance our understanding of the molecular-level processes that underlie the experimentally observed dynamics. They further served to highlight which aspects of the dynamics are correctly treated by analytical theories and which are not.

4. "Computer Simulations of Solvation Dynamics in Acetonitrile", $M$. Maroncelli, J. Chem. Phys. 94, 2084-2103 (1991)

Computer simulations of the solvation of monatomic ions in acetonitrile are used to investigate dynamical aspects of solvation in polar aprotic solvents. The observed dynarnics depend significantly on solute charge and on the which multipole moment of the solute is perturbed. In all cases, the solvation response has a two-part character. One part consists of a fast initial relaxation and attendant oscillations, both of which occur on a time scale of 0.1-0.2 ps. The initial response is well fit by a Gaussian function and accounts for $\sim 80 \%$ of the total relaxation. The second dynamical component occurs on a much slower, $\sim 1$ ps time scale, and accounts for the remainder of the relaxation. The fast response results from small amplitude inertial dynamics of solvent molecules within the confines of their instantaneous environment. The slow component reflects larger amplitude motions involving the breakup and reorganization of these local environments, especially in the first solvation shell of the solute. Comparison of the observed solvation dynamics to predictions of available theories points out the inadequacy of the latter due to their neglect of inertial parts of the solvent dielectric response.

9. "Simulations of Solvation in a Brownian Dipole Lattice", A. Papazyan and M. Maroncelli, J. Chem. Pliys. 95, 0000 (1991)

We have simulated the solvation of ions in a solvent consisting of point dipoles which undergo diffusive reorientation while translationally fixed to the sites of a cubic lattice. The simplicity of this model allows us to thoroughly explore how the energetics and dynamics of solvation depend on factors such as solute charge, solvent polarity, and number of solvent molecules. Some of the main features observed are as follows. The orientational response of first solvation shell dipoles saturates for moderate solute charges, resulting in a non-linear dependence of the 
reaction potential on solute charge. This non-linearity is to a good approximation independent of solvent polarity, and can be rationalized on the basis of a simple phenomenological model. One effect of the non-linear solvent response is to cause solvation free energy wells of the sort considered in electron transfer theories to be significantly anharmonic. Surprisingly, this deviation from harmonic behavior has little apparent impact on solvation barriers to charge transfer. The time-dependence of the solvation response deviates substantially from exponential behavior in the more polar systems studied. Solvation times (1/e times of the solvation response) are directly related to the magnitudes of fluctuations in the solvation potential. The dynamics of solvation for times $\leq t_{1 / \mathfrak{e}}$ can therefore be understood in terms of purely static correlations between solvent molecules. Dynamical inter-particle correlations are only important in determining the longer time behavior of the solvation response. In contrast to the long-ranged character of the solvation energy, only 20-30 solvent molecules are required to produce solvation times characteristic of bulk solvent.

We have begun several new lines of research only briefly mentioned in our original proposal. One of these involves studies of excited-state proton-transfer reactions that are catalyzed by alcohol solvents. Reference 7 is our first paper on the subject, and deals with the molecule 7-azaindole. The tautomerization of 7-azaindole solvents is controlled by a configurational rearrangement of solvent molecules directly hydrogen bonded to the solute. The observed reaction kinetics turn out to be a direct reflection of the dynamics of local hydrogen bond rearrangements in the immediate neighborhood of the solute. Such dynamics occur on a very different time scale from solvent energy relaxation so that this reaction probes a distinct new aspect of the solvent response.

7. "7.Axaindole in Alcohols: Solvation Dynamics and Proton Transfer", R. S. Moog and M. Maroncelli, J. Phys. Chem 95, 10359-10308 (199I).

The photoinduced excited state double proton transfer reaction of 7-azaindole has been examined in a variety of alcohol solvents. The influence of temperature and solvent deuteration has been investigated. A time-dependent Stokes shift of the initially excited norrnal species is observed, and this species is found to be the kinetic precursor to the tautomeric form. A substantial overlap of the normal and tautomer emissions is found, indicating that the tautomer emission must be monitored at wavelengths of $550 \mathrm{~nm}$ or greater to avoid contamination from the normal emission. The observed proton transfer times in normal alcohols at room temperature are well correlated with the solvation parameter $\mathrm{E}_{\mathrm{T}}(30)$, suggesting that the rate of proton transfer is related to the strength of solvent-solute interactions. However, an unusual temperature dependent isotope effect is also observed, in which the relative rates of proton transfer in norrnal and deuterated alcohols become closer as the temperature is lowered. These results are interpreted in terms of a two-step model fro proton transfer, involving solvent rearrangement to an appropriate configuration for the reaction to occur, followed by rapid proton transfer.

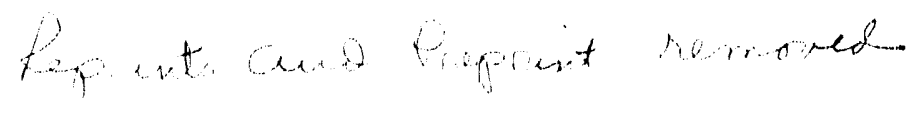




$$
\begin{aligned}
& \text { DATE } \\
& \text { FILMED } \\
& 813 / 192
\end{aligned}
$$


